\title{
Evidence-based knowledge on the aesthetics and maintenance of peri-implant soft tissues: Osteology Foundation Consensus Report Part 2-Effects of hard tissue augmentation procedures on the maintenance of peri-implant tissues
}

\author{
Frank Schwarz $^{1,2}$ | William V. Giannobile ${ }^{3}$ | Ronald E. Jung ${ }^{4}$ (D) on behalf of the \\ Groups of the 2nd Osteology Foundation Consensus Meeting
}

${ }^{1}$ Department of Oral

Surgery, Universitätsklinikum Düsseldorf,

Düsseldorf, Germany

${ }^{2}$ Department of Oral Surgery and Implantology, Carolinum, Johann Wolfgang Goethe-University Frankfurt, Frankfurt, Germany

${ }^{3}$ Department of Periodontics and Oral Medicine, University of Michigan School of Dentistry, Ann Arbor, MI, USA

${ }^{4}$ Clinic of Fixed and Removable Prosthodontics and Dental Material Science, University of Zurich, Zurich, Switzerland

\section{Correspondence}

Frank Schwarz, Department of Oral Surgery and Implantology, Johann Wolfgang GoetheUniversity Frankfurt, Frankfurt, Germany.

Email: info@frank-schwarz.com

Funding information

This Consensus Meeting was supported by a grant of the Osteology Foundation, Lucerne, Switzerland.

\begin{abstract}
Objectives: One task of Working Group 1 at the 2nd Consensus Meeting of the Osteology Foundation was to comprehensively assess the effects of hard tissue augmentation procedures on peri-implant health or disease.

Materials and methods: One systematic review and meta-analysis on the effects of hard tissue augmentation procedures included a total of eight studies ( $n=12$ publications). Consensus statements, clinical recommendations, and implications for future research were based on structured group discussions and plenary session approval. Results: After 1-10 years of follow- up, lateral bone augmentation procedures were associated with peri-implant tissue stability, as evidenced by minimal and non-significant changes in bleeding on probing, probing depth, and marginal bone levels. Case definitions based on clinical and radiographic parameters to differentiate peri-implant health from disease have been inconsistently employed in the studies investigated.

Conclusions: Lateral bone augmentation procedures are associated with peri-implant tissue stability on short-term (1-3 years) and midterm follow-ups to long-term (>3 years) follow-ups.
\end{abstract}

KEYWORDS

alveolar ridge augmentation, dental implant, peri-implant diseases

\section{1 | INTRODUCTION}

\begin{abstract}
Lateral ridge augmentation employing either autogenous bone or a variety of different bone replacement grafts has become an integral component of implant dentistry and was proven to be effective for the rehabilitation of both non-self-contained and self-contained osseous defects. In the latter situation, the outcome of therapy (i.e., percentage defect fill) was improved by the combined application of a barrier membrane (Sanz-Sanchez, Ortiz-Vigon, Sanz-Martin, Figuero \& Sanz, 2015).
\end{abstract}

While the implant survival rate at augmented sites was basically within the range of those data noted for pristine sites (Donos, Mardas \& Chadha, 2008), it was also assumed that ridge augmentation may constitute a potential risk indicator for peri-implant diseases (Canullo et al., 2016; Schwarz, Sahm \& Becker, 2012).

Therefore, one task of Working Group 1 of the Osteology Foundation Consensus Meeting was to comprehensively assess the effects of hard tissue augmentation procedures on peri-implant health or disease. 


\section{I WORKSHOP DISCUSSION AND CONSENSUS}

The present part 2 of the Osteology Foundation Consensus Report was based on the following systematic review:

1. Effects of lateral bone augmentation procedures on peri-implant health or disease-a systematic review and meta-analysis (SanzSanchez et al., 2017).

At the beginning of the meeting, the authors presented the systematic review in detail (i.e., methodology, results, conclusions) to the participants. Subsequently, the participants were separated into two working groups (Group 1: Maintenance of peri-implant soft tissues; Group 2: Aesthetics of peri-implant soft tissues). Discussions and the formulation of consensus statements within groups were each directed by one chairperson and one secretary. The statements, elaborated by the members of the working groups, were presented and discussed in plenary sessions and revised according to the suggestions made by the audience. Finally, consensus statements, clinical recommendations, and implications for future research were approved.

\subsection{Effects of lateral bone augmentation procedures on peri-implant health or disease-A systematic review and meta-analysis (Sanz-Sanchez et al., 2017)}

\subsection{1 | Focused question}

In situations with horizontal alveolar ridge deficiencies (population), what is the short- and long-term effect of different bone regenerative surgical interventions (intervention and comparison) on peri-implant health or disease (outcome)?

\subsection{2 | Major findings}

Lateral bone augmentation procedures are associated with peri-implant tissue stability based on bleeding on probing (BOP), probing depth (PD), and marginal bone levels (mBL) (ranging from 1 to 10 years of follow-up):

1. The results from the meta-analysis indicated that within each treatment approach, the changes in BOP over time are minimal and non-significant $[n=10 ; \mathrm{WMD}=-10.02 \% ; 95 \% \mathrm{Cl}(-22.23$ 2.21); $p=.108$ ]. When comparing different treatment modalities, no significant differences are observed $[n=6 ; \mathrm{WMD}=-3.36 \%$; 95\% Cl (-12.49; 5.77); $p<.471]$.

2. Similarly, changes in $P D$ and $m B L$ values are not significantly different among groups.

\subsection{3 | Consensus statements}

1. Case definitions based on clinical and radiographic parameters to differentiate peri-implant health from disease have been inconsistently employed in the studies investigated on lateral bone augmentation procedures.

2. Lateral bone augmentation procedures are associated with periimplant tissue stability based on BOP, PD, and $\mathrm{mBL}$.

3. Simultaneous and staged approaches to achieve defect fill or gain in ridge width presented a large heterogeneity with regard to types of interventions (i.e., particulate grafts of autogenous, xenogeneic, alloplastic origin; native/cross-linked and synthetic barrier membranes; intraoral autogenous and allogenic bone blocks, combinations with biological factors (i.e., rhBMP-2, rhPDGF-BB), or spontaneous healing).

4. The majority of the investigations employed a particulate deproteinized bovine bone mineral plus a native collagen membrane.

5. The presented meta-analysis has pointed to stable clinical and radiographic parameters at short-term (1-3 years) and midterm follow-ups to long-term (>3 years) follow-ups and failed to identify any significant differences between the interventions.

6. The occurrence of either peri-implant diseases or a progressive marginal bone loss was reported to be low and also comparable between test and control groups.

\section{2 | Clinical recommendations}

1. At bone deficient sites, the clinician may choose among different lateral bone augmentation procedures to develop implant sites in order to facilitate to maintain health and stability of peri-implant tissues.

2. When performing simultaneous bone grafting and implant placement, the emphasis of the procedure should be put on optimizing defect fill, as an incomplete defect fill (residual defects) increases the risk for peri-implant mucosal inflammation and progressive bone loss.

\section{3 | Implications for future research}

Future studies evaluating lateral bone augmentation procedures to treat bone defects at implant sites should focus on:

1. Using accepted case definitions in terms of peri-implant health and disease.

2. Investigating whether specific bone augmentation procedures provide comparable results to implants placed in native bone regarding tissue health and stability.

3. Investigating to what extent a deficient implant site may require grafting to maintain peri-implant health.

4. Analyzing other relevant endpoints, such as patient-reported outcome measures (PROMS)

5. Evaluating complementary diagnostic parameters to more precisely evaluate inflammatory states, such as the analysis of biomarkers. 
6. Evaluating the effect that local and systemic factors, such as history of periodontitis, smoking and uncontrolled diabetes, and other medications, may have on the outcomes of interest.

\section{CONFLICT OF INTEREST}

The authors and members of the working groups declare that they have no conflict of interests related to this consensus report. Frank Schwarz, William V. Giannobile and Ronald E. Jung are members of the Osteology Foundation Board

\section{ORCID}

William V. Giannobile iD http://orcid.org/0000-0002-7102-9746

Ronald E. Jung (iD http://orcid.org/0000-0003-2055-1320

\section{REFERENCES}

Canullo, L., Penarrocha-Oltra, D., Covani, U., Botticelli, D., Serino, G., \& Penarrocha, M. (2016). Clinical and microbiological findings in patients with peri-implantitis: A cross-sectional study. Clinical Oral Implants Research, 27, 376-382. https://doi.org/10.1111/clr.12557

Donos, N., Mardas, N., \& Chadha, V. (2008). Clinical outcomes of implants following lateral bone augmentation: Systematic assessment of available options (barrier membranes, bone grafts, split osteotomy). Journal of Clinical Periodontology, 35, 173-202. https://doi. org/10.1111/j.1600-051X.2008.01269.x

Sanz-Sánchez, I., Carrillo de Albornoz, A., Figuero, E., Schwarz, F., Jung, R. E., Sanz, M., \& Thoma, D. (2017). Effects of lateral bone augmentation procedures on peri-implant health or disease: A systematic review and meta-analysis. Clinical Oral Implants Research, 29(Suppl. 15), 18-31.

Sanz-Sánchez, I., Ortiz-Vigón, A., Sanz-Martín, I., Figuero, E., \& Sanz, M. (2015). Effectiveness of lateral bone augmentation on the alveolar crest dimension: A systematic review and metaanalysis. Journal of Dental Research, 94, 128S-142S. https://doi. org/10.1177/0022034515594780

Schwarz, F., Sahm, N., \& Becker, J. (2012). Impact of the outcome of guided bone regeneration in dehiscence-type defects on the long-term stability of peri-implant health: Clinical observations at 4 years. Clinical Oral Implants Research, 23, 191-196. https://doi. org/10.1111/j.1600-0501.2011.02214.x

How to cite this article: Schwarz F, Giannobile WV, Jung RE; on behalf of the Groups of the 2nd Consensus Meeting. Evidencebased knowledge on the aesthetics and maintenance of peri-implant soft tissues: Osteology Foundation Consensus Report Part 2-Effects of hard tissue augmentation procedures on the maintenance of peri-implant tissues. Clin Oral Impl Res. 2018;29(Suppl. 15):11-13. https://doi.org/10.1111/clr.13109 